\title{
Primary large cell neuroendocrine carcinoma of the common hepatic duct mimicking a Klatskin
} tumor

\author{
Ji Yeon Koo, Kook Hyun Kim, and Tae Nyeun Kim
}

Division of Gastroenterology and Hepatology, Department of Internal Medicine, Yeungnam University College of Medicine, Daegu, Korea

Received: May 24, 2017

Revised : August 3, 2017

Accepted: September 6, 2017

\section{Correspondence to}

Tae Nyeun Kim, M.D.

Tel: $+82-53-620-3842$

Fax: $+82-53-654-8386$

E-mail: tnkim@yu.ac.kr
A 77-year-old woman was admitted to Yeungnam University Hospital with jaundice and abdominal pain. Laboratory findings were as follows: total bilirubin, 9.0 mg/dL; aspartate aminotransferase, 199 IU/L; alanine aminotransferase, $120 \mathrm{U} / \mathrm{L}$; alkaline phosphatase, $1,592 \mathrm{IU} / \mathrm{L} ; \gamma$-glutamyl transferase, $937 \mathrm{IU} / \mathrm{L}$; and cancer antigen 19-9, 961 $\mathrm{U} / \mathrm{mL}$. An abdominal computed tomography (CT) scan revealed a 1-cmsized low-density mass in the perihilar area, with diffuse dilatation of the bilateral intrahepatic duct (Fig. 1C). Magnetic resonance cholangiopancreatography (MRCP) revealed a biliary stricture at the hepatic hilum, suggesting a Klatskin tumor (Fig. 1A). Endoscopic retrograde cholangiopancreatography (ERCP) was performed, and the findings were similar to those of MRCP
(Fig. 1B). Following an endobiliary forceps biopsy, a nasobiliary drain catheter was inserted. The tumor stained positive for $\mathrm{CD}_{5} 6$, synaptophysin, and chromogranin (Fig. 2). Approximately $60 \%$ of cells were positive for Ki67 (Fig. 2D). These were compatible with a large-cell neuroendocrine carcinoma (LCNEC). The patient refused further treatment and was discharged following the placement of a 10 Fr 12-cm biliary plastic stent. One month later, she was re-admitted with abdominal pain. CT showed multiple new metastases in the liver and lymph nodes (Fig. 1D). She underwent $30 \mathrm{~Gy} / 10$ Fr palliative radiotherapy for 2 weeks. Despite radiotherapy, her condition deteriorated, and she was transferred to a nursing home for supportive care. We received informed consent from the patient.
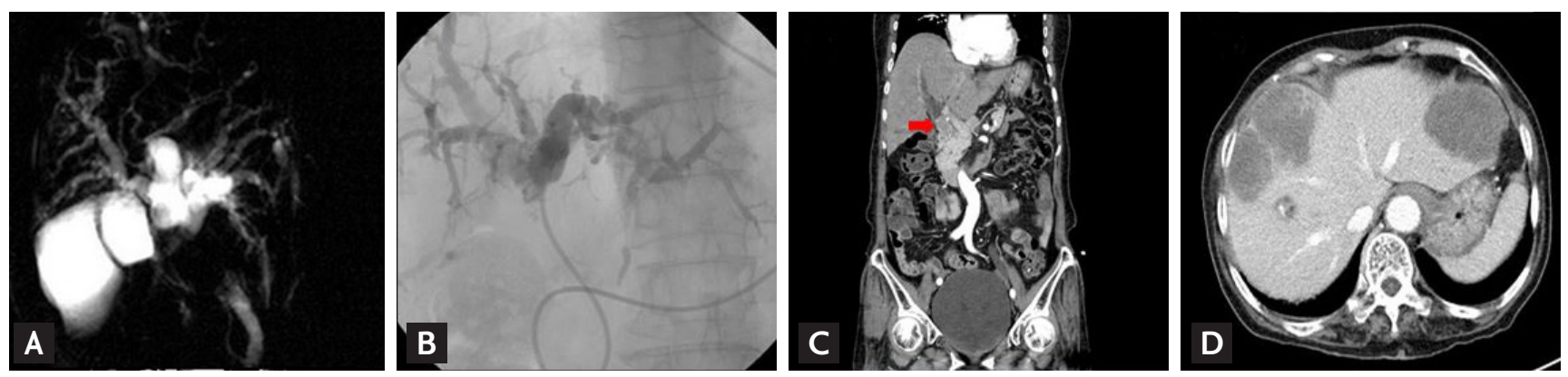

Figure 1. (A) Magnetic resonance cholangiopancreatography and (B) a cholangiogram via a nasobiliary drainage catheter, demonstrating a hilar biliary stricture, with dilation of both intrahepatic bile ducts. (C) Abdominal computed tomography (CT) revealing a $1 \mathrm{~cm}$, low-density mass in the perihilar area (arrow), with diffuse dilatation of the bilateral intrahepatic duct. (D)A CT scan obtained 1 month after the diagnosis, showing multiple metastases in both hepatic lobes. 

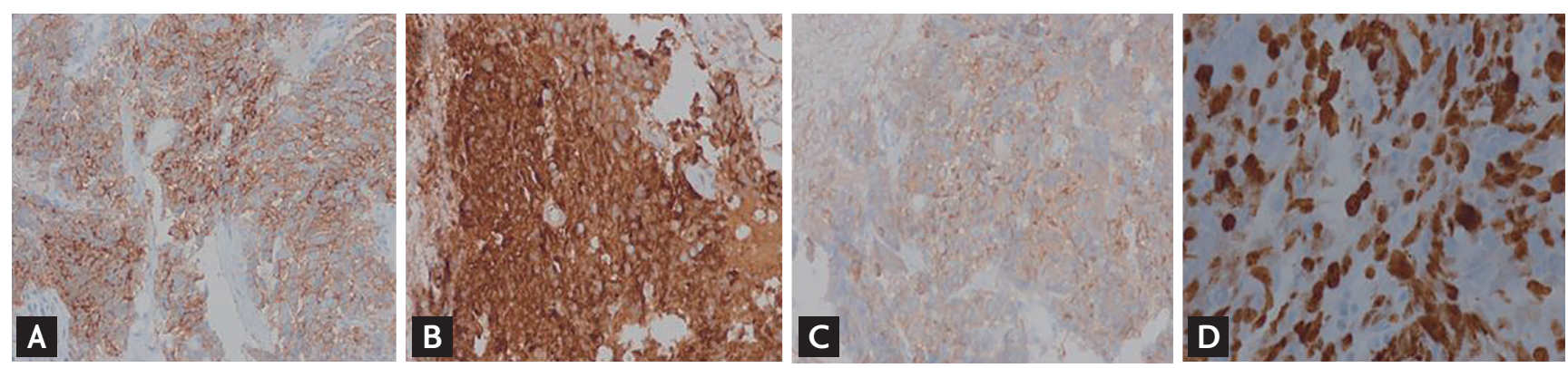

Figure 2. Immunohistochemical staining, showing that the tumor cells were strongly positive for (A) CD56 ( 2200$)$, (B) chromogranin ( $\times 200)$, and (C) synaptophysin (×200). (D) Approximately $60 \%$ of cells were positive for Ki-67 (×400).

LCNECs of the extrahepatic ducts are rare, with only four reported cases in the English literature. An LCNEC of the bile duct is an aggressive neoplasm. In the present case, extensive liver and lymph node metastases developed within 1 month of the diagnosis. A preoperative diagnosis of an LCNEC is challenging because of the difference in a biliary LCNEC on imaging. This is the first report of a diagnosis based on an endoscopic forceps biopsy sample obtained during ERCP. The biologi- cal characteristics of LCNECs are unknown. There is no definite treatment strategy, such as chemoradiotherapy or resection. Treatment options need to be studied in more detail.

\section{Conflict of interest}

No potential conflict of interest relevant to this article was reported. 